\title{
Brief report: Characteristics of antidepressant use in patients with heart failure
}

\author{
Paul J Mills' \\ Joel E Dimsdale' \\ Suzi Hong' \\ Geoffrey Van Den Brande 2 \\ Laura Redwine ${ }^{2}$ \\ Barry H Greenberg² \\ Thomas Rutledge' \\ 'Department of Psychiatry; \\ ${ }^{2}$ Department of Medicine, University \\ of California San Diego, La Jolla, \\ CA, USA
}

Background: Depression is common in heart failure (HF), but there is little data on the characteristics of antidepressant use in patients with HF.

Objective: To survey basic information on antidepressant prescription characteristics, use, effectiveness, and follow-up.

Methods: Observational study in two outpatient cardiology clinics of 37 NYHA class I-IV HF patients taking antidepressant medication.

Results: Thirty-one percent of prescriptions for antidepressants were obtained from psychiatrists, $58 \%$ from primary care physicians, and $8 \%$ from cardiologists. The majority of patients $(87 \%)$ reported regularly taking their antidepressant medication as prescribed, however $48 \%$ reported never having had the dosage of their antidepressant medication adjusted. Only $53 \%$ of the patients reported that the medication had helped their mood "almost entirely" or "mostly" back to normal since starting their antidepressants, while the remaining patients reported that their mood was only "halfway" or "somewhat" back to normal or that the medication had not helped their depression at all. Among a subset of 10 patients who completed the Beck Depression (BDI) inventory, 6 still had depressed $\operatorname{mood}(\mathrm{BDI} \geq 10)$.

Conclusion: The findings from this survey study provide insight into the characteristics of antidepressant use in patients with HF and argue for better follow up of HF patients who are prescribed antidepressants.

Keywords: heart failure, antidepressant medication, adherence, effectiveness

\section{Introduction}

Among patients with chronic illness, comorbid depression (depression superimposed on chronic illness) incrementally worsens health and quality of life (Moussavi et al 2007). Increasing attention is being paid to the high rates of comorbid depression in patients with HF (Rutledge et al 2006). In patients with HF, comorbid depression is present in up to $42 \%$ of cases and is associated with increased morbidity and mortality (Jiang et al 2001; Norra et al 2008).

Historically, few depressed HF patients received antidepressant treatment, although this has begun to change (Jacob et al 2003; Norra et al 2008). Although the efficacy of treating depression in ultimately altering cardiac related outcomes in HF is not established (Shapiro 2007), recent studies do suggest that treatment with certain antidepressants - including selective serotonin reuptake inhibitors (SSRI's) and tricyclics - might help reduce clinically relevant underlying inflammatory processes in HF (Tousoulis et al 2008).

What is missing from the literature is information about the characteristics of antidepressant use in HF. A number of clinically relevant questions are currently unanswered that could help guide future intervention efforts. For example, among HF patients receiving antidepressants, when did their depression begin in relation to their HF diagnosis? From whom did they receive the medication treatment? How aggressively 
was their antidepressant treatment followed and adjusted? And how efficacious was the treatment in the course of routine clinical practice? While it is known that adherence to HF medication is often poor in these patients (Hauptman 2008), and that this may be partially explained by the presence of depression in some patients (Morgan et al 2006), there is little published information on adherence to antidepressants in depressed HF patients.

In response to the scarcity of information in the literature, we developed a brief questionnaire as a method for gathering information on antidepressants and their use in patients with HF. In this brief report, we offer preliminary findings in this regard.

\section{Methods}

Thirty-seven stable NYHA class I-IV HF patients (age range 30 to 90 years) were studied. Participants were recruited from either the UCSD Heart Failure Program or the Veterans Affairs Medical Center Coronary Care Program. This study on antidepressant medication, which is part of a larger ongoing project on depression in HF, was approved by the University of California, San Diego Institutional Review Board. All subjects gave informed written consent.

Outpatients were approached at the clinics and asked if they were taking antidepressant medication and, if so, would they be willing to complete a brief questionnaire about their history and experiences with depression and antidepressants. Patients were told that their results would be kept confidential. Approximately 45 patients were approached to participate.

The 22 item questionnaire was developed by a team of psychologists and psychiatrists who are experienced with mental health factors in HF populations. Primary information sought in the questionnaire included: 1) who had prescribed their antidepressant (Psychiatrist, Primary Care Physician, Cardiologist, Other), 2) whether they were formally diagnosed with depression before receiving their antidepressant, 3) how long they had been feeling blue or depressed before receiving antidepressant treatment, 4) whether their symptoms of depression appeared before or after receiving their HF diagnosis, 5) their current antidepressant medication and dosage, 6) whether they had any dosage adjustments of their antidepressant medication, 7) whether they took their antidepressant regularly and at the prescribed dosage (and if not, why not), 8) whether the antidepressant had improved their mood (entirely or almost entirely back to normal, mostly back to normal, halfway back to normal, or somewhat back to normal, or no improvement), and 9) whether they were currently participating in adjunctive psychotherapy or behavioural therapy.

Answers from the questionnaire were analyzed descriptively and by chi-square tests where appropriate.

\section{Results}

Characteristics of the 37 patients who agreed to participate are presented in Table 1. Fifty-five percent of the patients reported that they had depressive symptoms prior to receiving their HF diagnosis. Only $43 \%$ of the patients reported that they had been formally diagnosed with depression prior to receiving antidepressant medication. Patients reported that they had been "feeling depressed or blue" for an average of 7.5 years $(\mathrm{SD}=10.2)$ before receiving treatment.

Thirty-one percent of patients reported that they received their antidepressants through a psychiatrist. Another 58\% reported that they received their antidepressants through their primary care physician, while three patients $(8 \%)$ reported receiving their antidepressant through his/her cardiologist.

The majority of patients (78\%) were on SSRI's (paroxetine, fluoxetine, citalopram, escitalopram, sertraline), with other medications including imipramine, mirtazapine, chlordiazepoxide and bupropion. Types of medications prescribed by psychiatrists versus primary care physicians did not differ significantly. Medication dosages provided by the patients were in line with standard prescriptions for these compounds.

Since receiving their prescription, nearly half (48\%) of the patients reported that they never had the dosage of their antidepressant medication adjusted. Eighty-seven percent reported that they regularly took their medication as prescribed. Seventy-three percent of patients receiving their medications from a psychiatrist had their dosages adjusted at least once, while only $42 \%$ of patients receiving their medications from a primary care physician had their dosages adjusted at least once (this difference was not statistically significant).

As far as effects of the antidepressants on mood, only $53.3 \%$ of the patients reported that the medication

Table I Sample characteristics (mean \pm SD)

\begin{tabular}{ll}
\hline Sample size & 37 \\
Age (years) & $6 I .6( \pm 35)$ \\
Gender & 29 men; 8 women \\
NYHA Class (available on 34 & $\mathrm{I}-8$ \\
of the 37 patients) & $\mathrm{II}-\mathrm{I} 4$ \\
& $\mathrm{III}-7$ \\
& $\mathrm{IV}-5$ \\
Ejection fraction (\%) & $40.8( \pm \mathrm{I2})$ \\
\hline
\end{tabular}


had helped their mood to get "almost entirely" or "mostly" back to normal, while $36.6 \%$ reported that their mood was only "halfway" or "somewhat" back to normal, and $10 \%$ reported that the medication had not helped their mood at all (Figure 1). There were no differences in reported mood between patients who had their depressive symptoms prior to versus after receiving their HF diagnosis, or between those who had versus never had their dosage adjusted.

At the time of completing the 22-item questionnaire, as part of another larger study, 10 of the patients also completed the Beck Depression Inventory (BDI) (Beck et al 1996). Six of these patients still had elevated depressive mood (BDI $\geq 10$ ) (mean score 16.4; range 10 to 34 ) and 4 of those could be considered clinically depressed (BDI $\geq 17$ ) (mean score 26.7 , range 17 to 34 ).

Twenty percent of the patients reported currently participating in adjunctive/behavioral therapy.

\section{Discussion}

Depression remains a highly important topic in HF. Prevalence rates of clinically significant depression in HF range from $11 \%$ to $42 \%$ among New York Heart Association classes I versus IV, respectively, with overall estimates of approximately 30\% (Rutledge et al 2006; Norra et al 2008). Recent reviews of the HF literature demonstrate that comorbidity with depression is associated with increased healthcare utilization, functional disability, and a poorer medical prognosis (Rutledge et al 2006; Egede 2007).
It is known that only a small percentage of HF patients with comorbid depression receive antidepressant medication (Norra et al 2008). Our study was not designed to provide an estimate of prevalence of antidepressant usage, but rather information about patients who have received such medication. We are unaware of any study to date that has described characteristics of antidepressant use among HF patients being treated for depression. Although adherence to HF medication is reported as often poor in patients with HF (Hauptman 2008), the majority (87\%) in our sample reported that they regularly took their antidepressant medication as prescribed, although only roughly half reported that their mood had returned to normal or near normal.

Only three of the patients reported receiving antidepressant medication from their cardiologist, with the remainder receiving them from a psychiatrist or primary care physician. The majority of patients were on selective serotonin reuptake inhibitors, which are considered effective therapy for HF patients with depression (Parissis et al 2007; Serebruany et al 2003).

Recent studies find that among HF patients at different stages of their illness, changes over time in depression symptoms are important to clinical outcomes (Koenig et al 2006; Azevedo et al 2008). Among the important findings from this survey include that depressed HF patients on antidepressant medication are not being routinely followed to optimize therapy. Half of the patients in this study reported never having had the dosage of

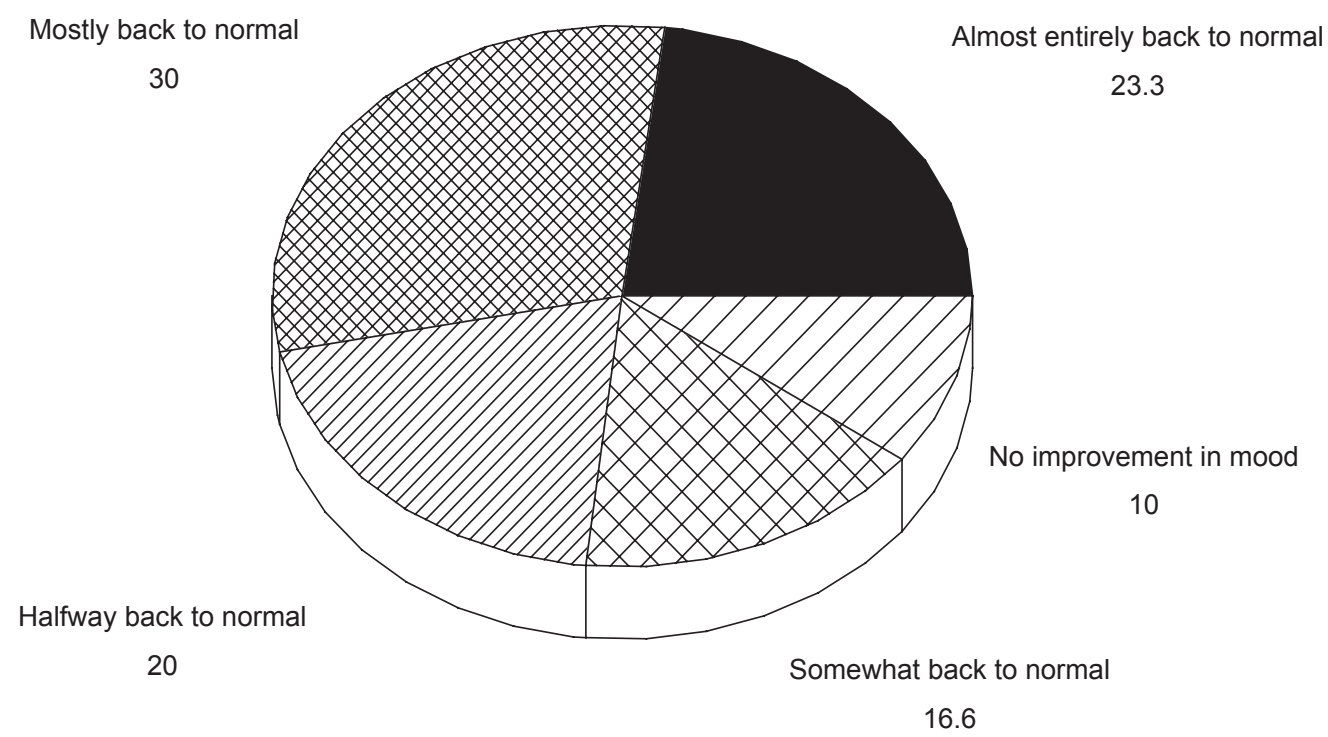

Figure I The figure presents the respective percentages of patients providing one of five possible responses to the question, “Do you think the antidepressant medication has improved your mood?' Patients could respond with either my mood is 'Almost entirely back to normal','Mostly back to normal','Halfway back to normal','Somewhat back to normal', or 'No improvement'. Only slightly more than half of the patients $(53.3 \%)$ reported a significant improvement in their depression. 
their antidepressant medication adjusted and a significant percentage self reported that their mood was not back to normal (Adams et al 2008). A partial response to antidepressant treatment is known to be a strong predictor of depression relapse (Furukawa et al 2007). Among the subset of patients on antidepressants who completed the BDI, 60\% still had depressed mood, which is consistent with the reports from the larger cohort of roughly $46 \%$ reporting that their mood had not returned to normal or mostly normal.

We designed this questionnaire in response to what we perceived as gaps in the literature on HF and antidepressant use. We do not know the degree to which our findings might generalize to other HF patients taking antidepressants or how the findings in this sample might differ from those patients at our clinics who refused to participate in this study. Approximately $85 \%$ of those patients approached were willing to complete the questionnaire, which is a considerably higher percentage compared to a $49 \%$ willingness to participate rate in a study of attitudes about impairment in HF patients prescreened for depression (Turvey et al 2006).

The modest sample size is a limitation of this study. This is an ongoing project, however, and it is our intention to expand the sample size and scope of this particular line of research. In addition, we did not gather information on family history of depression, which does have a bearing on depression treatment and clinical course in HF (Artinian et al 2004; Koenig et al 2006).

In summary, these findings provide some preliminary insight into the characteristics of antidepressant use in patients with HF. Despite receiving antidepressant treatment, results from our sample suggest that many HF patients are inadequately treated and continue to experience depressed mood and may be vulnerable to relapse or progression of their depression, particularly as their HF advances. Similarly, the aggressiveness of treatment follow-up and dosage adjustments for antidepressant therapy are less than optimal for achieving long term improvement in depressive symptoms. Although the importance of treating depression in $\mathrm{HF}$ is receiving increasing recognition (Blier et al 2007; Shapiro 2007), our findings indicate that attention to the process and delivery of antidepressant treatment for these patients needs considerably more attention.

\section{Disclosure}

The authors report no conflicts of interest in this work.

\section{Acknowledgments}

This work was supported by grants HL-073355 and HL-57265 from the NIH.

\section{References}

Adams SM, Miller KE, Zylstra RG. 2008. Pharmacologic management of adult depression. Am Fam Physician, 77:785-92.

Artinian NT, Artinian CG, Saunders MM. 2004. Identifying and treating depression in patients with heart failure. $J$ Cardiovasc Nurs, 19(Suppl 6):S47-56.

Azevedo A, Bettencourt P, Frioes F, et al. 2008. Depressive symptoms and heart failure stages. Psychosomatics, 49:42-8.

Beck AT, Steer RA, Brown GK. 1996. Beck Depression Inventory, 2nd ed. Manual. San Antonio, Tx: Psychological Corp.

Blier P, Keller MB, Pollack MH, et al. 2007. Preventing recurrent depression: long-term treatment for major depressive disorder. J Clin Psychiatry, 68:e06.

Egede LE. 2007. Major depression in individuals with chronic medical disorders: prevalence, correlates and association with health resource utilization, lost productivity and functional disability. Gen Hosp Psychiatry, 29:409-16.

Furukawa TA, Cipriani A, Barbui C, et al. 2007. Long-term treatment of depression with antidepressants: a systematic narrative review. Can $J$ Psychiatry, 52:545-52.

Hauptman PJ. 2008. Medication adherence in heart failure. Heart Fail Rev, 13:99-106.

Jacob S, Sebastian JC, Abraham G. 2003. Depression and congestive heart failure: are antidepressants underutilized? Eur J Heart Fail, 5:399-400.

Jiang W, Alexander J, Christopher E, et al. 2001. Relationship of depression to increased risk of mortality and rehospitalization in patients with congestive heart failure. Arch Intern Med, 161:1849-56.

Koenig HG, Johnson JL, Peterson BL. 2006. Major depression and physical illness trajectories in heart failure and pulmonary disease. J Nerv Ment Dis, 194:909-16.

Koenig HG, Vandermeer J, Chambers A, et al. 2006. Minor depression and physical outcome trajectories in heart failure and pulmonary disease. J Nerv Ment Dis, 194:209-17.

Morgan AL, Masoudi FA, Havranek EP, et al. 2006. Difficulty taking medications, depression, and health status in heart failure patients. $J$ Card Fail, 12:54-60.

Moussavi S, Chatterji S, Verdes E, et al. 2007. Depression, chronic diseases, and decrements in health: results from the World Health Surveys. Lancet, 370:851-8.

Norra C, Skobel EC, Arndt M, et al. 2008. High impact of depression in heart failure: Early diagnosis and treatment options. Int J Cardiol, 125:220-31.

Parissis J, Fountoulaki K, Paraskevaidis I, et al. 2007. Sertraline for the treatment of depression in coronary artery disease and heart failure. Expert Opin Pharmacother, 8:1529-37.

Rutledge T, Reis VA, Linke SE, et al. 2006. Depression in heart failure a meta-analytic review of prevalence, intervention effects, and associations with clinical outcomes. J Am Coll Cardiol, 48:1527-37.

Serebruany VL, Glassman AH, Malinin AI, et al. 2003. Selective serotonin reuptake inhibitors yield additional antiplatelet protection in patients with congestive heart failure treated with antecedent aspirin. Eur J Heart Fail, 5:517-21.

Shapiro PA. 2007. Treatment of depression in patients with congestive heart failure. Heart Fail Rev, Oct 23. [Epub ahead of print].

Tousoulis D, Drolias A, Antoniades C, et al. 2008. Antidepressive treatment as a modulator of inflammatory process in patients with heart failure: Effects on proinflammatory cytokines and acute phase protein levels. Int $J$ Cardiol, Jun 23. [Epub ahead of print].

Turvey CL, Klein DM, Pies CJ. 2006. Depression, physical impairment, and treatment of depression in chronic heart failure. $J$ Cardiovasc Nurs, 21:178-85. 